\title{
SKA2 Methylation is Involved in Cortisol Stress Reactivity and Predicts the Development of Post-Traumatic Stress Disorder (PTSD) After Military Deployment
}

\author{
Marco P Boks*, , Bart PF Rutten ${ }^{2}$, Elbert Geuze ${ }^{1,3}$, Lotte C Houtepen', Eric Vermetten ${ }^{1,3,4,5}$, \\ Zachary Kaminsky,7 and Christiaan H Vinkers' \\ 'Department of Psychiatry, Brain Center Rudolf Magnus, University Medical Centre Utrecht, Utrecht, The Netherlands; ${ }^{2}$ Department of Psychiatry \\ and Neuropsychology, School for Mental Health and Neuroscience, Maastricht University Medical Centre, Maastricht, The Netherlands; ${ }^{3}$ Research \\ Centre Military Mental Healthcare, Ministry of Defence, The Hague, The Netherlands; ${ }^{4}$ Department of Psychiatry, Leiden University Medical \\ Center, Utrecht, The Netherlands; ${ }^{5}$ Arq Psychotrauma Expert Group, Diemen, The Netherlands; ${ }^{6}$ Department of Psychiatry and Behavioral \\ Sciences, The Mood Disorders Center, Johns Hopkins School of Medicine, Baltimore, MD, USA; ${ }^{7}$ Department of Mental Health, Johns Hopkins \\ Bloomberg School of Public Health, Baltimore, MD, USA
}

Genomic variation in the SKA2 gene has recently been identified as a promising suicide biomarker. In light of its role in glucocorticoid receptor transactivation, we investigated whether SKA2 DNA methylation influences cortisol stress reactivity and is involved in the development of post-traumatic stress disorder (PTSD). Increased SKA2 methylation was significantly associated with lower cortisol stress reactivity in 85 healthy individuals exposed to the Trier Social Stress Test $(B=-173.40, t=-2.324$, $p$-value $=0.023)$. Next, we observed that longitudinal decreases in SKA2 methylation after deployment were associated with the emergence of post-deployment PTSD symptoms in a Dutch military cohort $\left(N=93 ; B=-0.054, t=-3.706, p\right.$-value $\left.=3.66 \times 10^{-4}\right)$. In contrast, exposure to traumatic stress during deployment by itself resulted in longitudinal increases in SKA2 methylation $\left(B=0.037, t=4.173, p\right.$-value $\left.=6.98 \times 10^{-5}\right)$. Using predeployment SKA2 methylation levels and childhood trauma exposure, we found that the previously published suicide prediction rule significantly predicted post-deployment PTSD symptoms (AUC $=0.66,95 \% \mathrm{Cl}: 0.53-0.79$ ) with an optimal sensitivity of $0.8 \mathrm{I}$ and specificity of 0.91 . Permutation analysis using random methylation loci supported these findings. Together, these data establish the importance of SKA2 for cortisol stress responsivity and the development of PTSD and provide further evidence that SKA2 is a promising biomarker for stress-related disorders including PTSD.

Neuropsychopharmacology (2016) 4I, I350-1356; doi:I0. I038/npp.20 I5.286; published online 7 October 2015

\section{INTRODUCTION}

Prevalence rates of post-traumatic stress disorder (PTSD) in military personnel following deployment range from 5 to 15\% (Sundin et al, 2010; Reijnen et al, 2015). Even though several risk factors for deployment-related PTSD have been identified (Sandweiss et al, 2011), the etiology of PTSD is currently not fully understood. It is therefore not possible to accurately identify who is at risk for PTSD after exposure to traumatic stress, and no reliable blood-based biomarkers for PTSD vulnerability have been identified so far. Nevertheless, prediction of deployment-related PTSD vulnerability is of great importance as it would facilitate prevention of the detrimental social and personal consequences of PTSD.

*Correspondence: Dr MPM Boks, Department of Psychiatry, Brain Center Rudolf Magnus, University Medical Centre Utrecht, HP. A.01.489, PO Box 85500, Utrecht, 3508 GA, The Netherlands, Tel: +3I 88 7556370, Fax: +31 88 7555509, E-mail: mboks@umcutrecht.nl Received 2 June 2015; revised 18 August 2015; accepted 28 August 2015; accepted article preview online II September 2015
Recent studies have shown that epigenetic mechanisms are important for successful adaptation to a stressful environment and have a role in the development of PTSD (Zovkic et al, 2013). DNA methylation is an important epigenetic mechanism that is influenced by genetic and environmental factors (Malan-Muller et al, 2014), and reflects the transcriptional status of a particular gene (Schubeler, 2015). DNA methylation studies therefore have great potential to increase our understanding of how the interaction between an individual's genetic background and traumatic stress exposure results in the development of PTSD (Vinkers et al, 2015a). Kaminsky and colleagues recently proposed the spindle and kinetochore associated protein 2 (SKA2) gene (Chr17:59110368) as a blood-based DNA methylation biomarker for suicide (Guintivano et al, 2014). In their study, SKA2 methylation levels predicted suicide and were also related to lower levels of the stress hormone cortisol, consistent with a role in glucocorticoid receptor transactivation (Rice et al, 2008). As PTSD is associated with enhanced GR sensitivity and lower physiological hypothalamic- 
pituitary-adrenal (HPA) axis activity (Mehta and Binder, 2012; De Kloet et al, 2006; Yehuda et al, 1991) as well as with higher suicide rates (Schoenbaum et al, 2014), these findings raise the question whether the epigenetic SKA2 biomarker can also be of use for detecting who is at risk for PTSD. We hypothesize that changes in SKA2 methylation are dissimilar between PTSD-susceptible individuals and those who are resilient.

To investigate the involvement of SKA2 methylation, we used data from two independent cohorts. First, to understand how SKA2 methylation levels are related to the endocrine adaptation to stress, we examined the relationship between SKA2 methylation and the cortisol stress response in healthy individuals. Second, we prospectively investigated changes in SKA2 methylation in relation to the development of PTSD symptoms using data from a longitudinal Dutch military cohort (Boks et al, 2015) in which blood-based DNA methylation levels of SKA2 were measured before and 6 months after deployment to Afghanistan. Third, we investigated whether the epigenetic SKA2 biomarker rule developed for suicide (Guintivano et al, 2014) is capable of predicting PTSD before deployment.

\section{MATERIALS AND METHODS}

\section{Samples}

Cortisol stress sample. Eighty-five healthy individuals were recruited from the general population at the University Medical Center, Utrecht, The Netherlands. Participants had three or more Dutch grandparents and had not been enrolled in stress-related research before (Vinkers et al, 2013; Houtepen et al, 2015; Table 1). The absence of any mental or physical disorder was confirmed by an independent rater. Participants did not take heavy meals, drinks other than water or carry out heavy exercise at least $2 \mathrm{~h}$ before the study protocol. Current use of psychoactive substances (amphetamines, MDMA, barbiturates, cannabinoids, benzodiazepines, cocaine, and opiates) was determined by self-report and verified with a urine multi-drug screening device (InstantView). Participants completed a group version of the Trier Social Stress Test (G-TSST), consisting of a public speaking test and an arithmetic task as previously published (Houtepen et al, 2015). Cortisol stress reactivity was assessed using eight saliva samples (Salivettes) collected over a time period of $90 \mathrm{~min}$ (Supplementary Figure S1). Samples were directly stored at $-80^{\circ} \mathrm{C}$ and analyzed as previously described (Houtepen et al, 2015). In short, cortisol was measured without extraction using an in house competitive

Table I Characteristics of the Cortisol Stress Reactivity Sample $(N=85)$

\begin{tabular}{lc} 
Characteristics & \\
\hline Sex (\% female) & 50.5 \\
Mean age (SD) & $33(15.84)$ \\
Race (\% European Caucasian) & 100 \\
Childhood trauma (mean and SD) & $31.9(8.2)$ \\
(mean AUCi, SD) & $242.3(419.64)$
\end{tabular}

radio-immunoassay. The area under the curve increase (AUCi) of cortisol was calculated based on the consecutive data points as described by Pruessner et al (2003).

Childhood trauma was assessed using the 25-item of the Childhood Trauma Questionnaire (CTQ; Bernstein et al, 2003). The validity of the CTQ, including a Dutch translation, has been demonstrated in clinical and community samples (Bernstein et al, 2003; Thombs et al, 2009). One translated item (I believe I was molested) was excluded as this translation was found to be an invalid indicator of childhood sexual abuse in a previous validation study (Thombs et al, 2009).

\section{Military Sample}

From a large prospective cohort of 1032 Dutch military personnel deployed to Afghanistan (Van Zuiden et al, 2011), three approximately equally sized male subgroups (total $N=94)$ were selected based on level of traumatic stress exposure and presence of PTSD symptoms (Table 2). In this selected sample we analyzed the association of longitudinal changes of SKA2 methylation with trauma exposure and PTSD symptom increase. Sample size was determined based on previous studies of cortisol response in healthy controls (cortisol stress sample). Blood samples were collected before and 6 months after deployment. Current PTSD symptoms over the past 4 weeks were assessed with the 22-item selfreport inventory for PTSD (SRIP) which has a good reliability and validity compared with other PTSD scales such as the Clinical Administered PTSD scale and the Mississippi scale for PTSD (Keane et al, 1988; Hovens et al, 2000). Development of PTSD was defined as a score of 38 and higher at follow-up in agreement with our previous studies (Van Zuiden et al, 2011). Exposure to combat trauma during deployment was assessed with a 19-item deployment experiences checklist (DEC) as previously published (Reijnen et al, 2015). This self-report assessment provided a range of potentially traumatic experiences that occur as part of deployment which include direct combat stressors. Trauma exposure was defined as $>1$ traumatic event. This pre-set dichotomization is based on the selection of participants with low or high levels of trauma to increase power (Boks et al, 2007) and leads to a bivariate distribution of trauma levels (see Supplementary Figure S4 for histogram).

After exclusion of one outlier (see next paragraph) 93 participants were analyzed: (i) $N=32$ with high combat trauma exposure (DEC, mean $=7.3, \mathrm{SD}=2.9)$ and high levels of post-deployment PTSD symptoms (SRIP, mean $=45.3$, $\mathrm{SD}=8.6$ ), (ii) $N=29$ with high combat trauma exposure $(\mathrm{DEC}$ mean $=8.6, \mathrm{SD}=2.3$ ) and low levels of PTSD symptoms ( $\mathrm{SRIP}=26.0, \mathrm{SD}=3.7$ ), and (iii) $N=32$ with low combat trauma exposure (DEC mean $=0.4, \mathrm{SD}=0.5)$ and low levels of post-deployment PTSD symptoms (SRIP mean $=25.1, \mathrm{SD}=3.7$; Table 2). Analysis of age, gender, alcohol consumption, cigarette smoking, military rank, length, weight, or medication use did not show any differences between these three groups. Childhood trauma was assessed with the 27-item Dutch version of the Early Trauma Inventory-Self Report (Bremner et al, 2007), assessing early traumatic experiences before the age of 18 years which include general trauma, physical abuse, emotional abuse, and sexual abuse (Hovens et al, 2000; 
Table 2 Demographic and Clinical Characteristics of the Military Sample

\begin{tabular}{|c|c|c|c|c|}
\hline Characteristics & All $(N=93)$ & PTSD $(N=32)$ & High trauma $(N=29)$ & Low trauma $(N=32)$ \\
\hline Post-deployment PTSD symptoms & & High & Low & Low \\
\hline Traumatic stress exposure during deployment & & High & High & Low \\
\hline Race (\% European Caucasian) & 100 & 100 & 100 & 100 \\
\hline Age (SD) & $27.5(9.1)$ & $26.8(9.6)$ & $27.7(9.1)$ & $28.1(8.8)$ \\
\hline Mean trauma score (SD) & $5.3(4.2)$ & $7.3(2.9)$ & $8.6(2.3)$ & $0.4(0.5)$ \\
\hline PTSD symptoms at follow-up (SD) & $32.4(11.1)$ & $45.3(8.6)$ & $26.0(3.7)$ & $25.1(3.7)$ \\
\hline Change PTSD symptoms (SD) & $5.9(10.8)$ & $17.4(10.1)$ & $0.0(3.6)$ & $-0.3(4.8)$ \\
\hline Change in SCL-90 (SD) & $6.6(21.8)$ & $20.4(31.0)$ & $0.8(11.5)$ & $0.7(10.1)$ \\
\hline Childhood trauma (SD) & $3.1(2.7)$ & $4.8(3.1)$ & $3.2(2.3)$ & $1.3(1.1)$ \\
\hline Number of deployment (SD) & $0.9(1.2)$ & $0.8(1.2)$ & $1.2(1.5)$ & $0.6(0.8)$ \\
\hline
\end{tabular}

Witteveen et al, 2006; Hovens et al, 2002; Witteveen et al, 2006). To investigate the specificity of changes in PTSD symptoms we also investigated SCL-90 total score (psychoneuroticism) as well as the depression, somatisation, agoraphobia, and anxiety subscales (Derogatis et al, 1973). Differences in PTSD symptoms between time points were log-transformed to improve the distribution. Three missing values in the baseline measure of PTSD symptoms were replaced by median values. This study was approved by the Medical Ethics Committee of the University Medical Center Utrecht and conducted in accordance with the Declaration of Helsinki and all participants gave written informed consent.

\section{SKA2 Genotype and Methylation}

In both the stress reactivity and the military sample, whole blood EDTA samples were obtained and DNA was extracted using standard salting procedures. DNA concentration was assessed using riboGreen and integrity using BioAnalyser. Bisulphite conversion was conducted using Zymo kits under standard protocol. In both samples, DNA methylation levels were assessed using Illumina 450K DNA methylation arrays interrogating over $450000 \mathrm{CpG}$ loci per sample at single-nucleotide resolution and covering 99\% of RefSeq genes and $96 \%$ of $\mathrm{CpG}$ islands. For the military sample, baseline and follow-up samples were positioned on the same array and exposure to trauma and PTSD outcomes were equally distributed over the 16 arrays to reduce any batch effects to the minimum. For all analyses, SKA2 methylation levels (cg13989295) were adjusted for age and genotype of the underlying SNP (rs7208505) using inference of genotype based on the distributions of methylation levels as previously employed (Guintivano et al, 2014). Analyses of our data showed that SKA2 genotypes completely separate methylation distributions (Supplementary Figure S2). Adjusted SKA2 methylation levels were derived by taking the residuals of a linear model with age and genotype as indicator. One outlier of SKA2 methylation levels, defined as more than three SD from the mean, was excluded from analyses.

\section{Cell-Type Composition}

Cell-type composition was assessed in light of its potential influence on DNA methylation. In the military sample, celltype composition was investigated using flow cytometry analysis as implemented in the clinical laboratory of our University Medical Center as previously published (Van Zuiden et al, 2009; Supplementary Figure S3). In the cortisol stress sample, cell-count estimation was calculated based on a recent algorithm (Jaffe and Irizarry, 2014). The effect of cell-count composition on SKA2 methylation levels was analyzed for both samples.

\section{Statistical Analysis}

In the cortisol stress sample, linear regression was carried out with SKA2 methylation as the main determinant of the stress-induced cortisol area under the curve $\left(\mathrm{AUC}_{\mathrm{i}}\right)$, age and gender and childhood trauma as covariates (cortiso1_AUCi SKA2 methylation+age+gender+childhood trauma). Mediation analysis was conducted using the mediate package in $R$ that implements a bootstrapping procedure to infer if changes in SKA2 methylation that are accounted for by childhood trauma can explain the association of childhood trauma with cortisol response. This effectively estimates the extent to which SKA2 methylation levels explain the association between childhood trauma and the cortisol stress response.

In the military sample, longitudinal analysis were conducted with SKA2 methylation after deployment as the outcome using linear regression with baseline SKA2 methylation levels as a covariate. First, we analyzed all participants together in a model with indicators of trauma and PTSD simultaneously (SKA2 methylation $_{\text {postdeployment }}$ SKA2 methylation predeployment+change in PTSD symptoms+trauma exposure).

Next, to further separate effects of trauma and PTSD on SKA2 methylation, stratified analyses were carried out after exclusion of either participants who were not exposed to trauma (investigating PTSD symptoms in the traumaexposed group) and excluding those individuals who 
developed post-deployment PTSD symptoms (investigating trauma effects in the non-PTSD group).

All models were analyzed, with and without childhood trauma and the interaction term of childhood trauma and traumatic stress. Permutations with 100000 random CpG methylation levels were used to derive an empirical $p$-value and estimate of the degree to which the association with traumatic stress and PTSD is unique or is an artifact of the underlying data structure. To investigate the effect of time we used a paired $t$-test to compare pre- and post-deployment SKA2 methylation levels.

To validate the previously published SKA2 suicide prediction rule (Guintivano et al, 2014) for its utility to predict PTSD, we used the model from the published training set in suicide victims on our data with the same published model parameters. The prediction rule was analyzed for performance using a model with PTSD as dependent and SKA2 methylation and genotype in interaction with premorbid stress (suicide $\sim$ (SKA2 methylation +genotype) $\times$ childhood trauma). $p$-Values for prediction were obtained using $1 \times 10^{5}$ label swapping permutations. This model was further validated using permutation analyses comparing the performance of an adjusted SKA2 model from the training set (using genotype and age-adjusted SKA2 methylation levels) with $1 \times 10^{5}$ random CpGs from the $450 \mathrm{~K}$ methylation array to derive an empirical $p$-value. In addition we created a new prediction rule for the prospective development of PTSD symptoms, which was investigated using ROC analyses with pre-deployment SKA2 methylation levels as indicators.

Assumptions of linear regression were evaluated by inspecting residuals. Cell-type composition before and after deployment was compared using repeated measures analysis of variance. Potential confounding by cell-type was ruled out by analyzing the association between cell counts (Monocytes, CD8T cells, CD4T cells, B-cell, and Natural Killer cells) with SKA2 methylation levels. In the cortisol stress sample, medication users and smokers were excluded. In the military sample, the potential influence of childhood trauma was investigated by adding continuous childhood trauma scores to the above mentioned models and by analyzing baseline differences between the PTSD and trauma groups using a one way analysis of variance (ANOVA). The potential influence of cigarette smoking, alcohol use, and medication was investigated by excluding participants with documented change in these parameters from the analysis $(N=35, N=8$, and $N=18$, respectively).

\section{RESULTS}

\section{SKA2 Methylation is Involved in Cortisol Stress Reactivity}

In 85 healthy individuals, a lower cortisol stress response was significantly associated with SKA2 methylation in a model including age, gender, and childhood trauma (Table 3; model fit: $\left.\mathrm{F}(4,84)=9.12, p<0.001, R^{2}=0.27\right)$. Mediation analysis showed no evidence for a causal role of SKA2 methylation in the relationship between childhood trauma and cortisol reactivity (estimated proportion mediation $=0.041 \%, 95 \%$ CI: $-0.18-0.233, p$-value $=0.52$ )
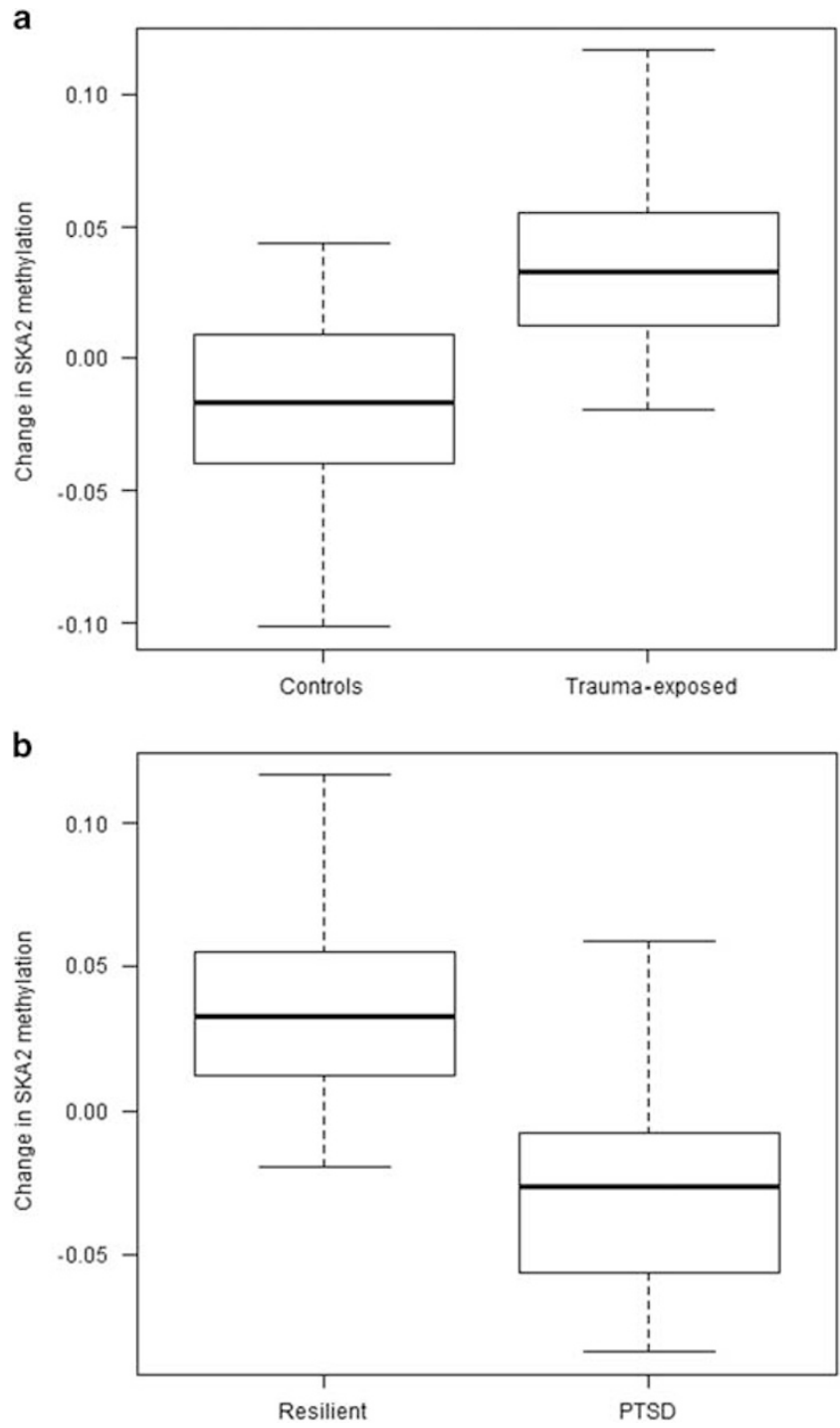

Figure I (a) SKA2 methylation changes during deployment in (non-posttraumatic stress disorder (PTSD)) individuals that were exposed to either low- or high-levels of trauma. In individuals exposed to traumatic stress, SKA2 methylation levels increases were significantly higher ( $p$-value $=9.5 \times$ $10^{-5}$ ). (b) SKA2 methylation changes during deployment in participants that developed PTSD symptoms and those resilient to trauma. In individuals who developed PTSD symptoms after deployment, SKA2 methylation increases were significantly lower $\left(p\right.$-value $\left.=6.7 \times 10^{-5}\right)$.

\section{Longitudinal Changes in SKA2 Methylation and the Association with Trauma and PTSD}

In the military cohort, we investigated the relationship between traumatic stress during deployment, longitudinal changes in SKA2 methylation and PTSD symptoms. Concurrent analysis of trauma and PTSD showed that SKA2 methylation changes were negatively associated with the development of PTSD symptoms $(B=-0.054, t=-3.706$, $p$-value $=3.66 \times 10^{-4}$, empirical $p$-value $\left.=1.37 \times 10^{-3}\right)$ and that trauma exposure during deployment was positively associated with change in SKA2 methylation $(B=0.037, t=4.173$, $p$-value $=7.0 \times 10^{-5}$, empirical $p$-value $\left.=6.4 \times 10^{-4}\right)$. Addition of childhood trauma as covariate retained a significant 
Table 3 Association of SKA2 Methylation with the Cortisol Stress Response in Healthy Controls

\begin{tabular}{lcccc}
\hline & B & SE & $\boldsymbol{t}$ & $\boldsymbol{p}$-value \\
\hline SKA2 methylation & -173.404 & 74.624 & -2.324 & 0.02256 \\
Childhood trauma & -21.433 & 6.619 & -3.238 & 0.00172 \\
Age & 3.955 & 2.529 & 1.564 & 0.12164 \\
Gender & -254.208 & 78.359 & -3.244 & 0.00169 \\
\hline
\end{tabular}

association of SKA2 methylation with PTSD symptoms $\left(B=-0.058, t=-3.880 p\right.$-value $\left.=2.0 \times 10^{-4}\right)$ and deploymentrelated traumatic stress $(B=0.032, \quad t=3.339, \quad p$-value $=$ $0.001235)$. Childhood trauma was not significantly associated with prospective changes in SKA2 methylation $(B=0.002$, $t=1.291, p$-value $=0.20$ ) nor did childhood trauma alter the effects of traumatic stress on SKA2 methylation ( $B=-0.001993$, $t=-0.325$, $p$-value $=0.75$ ). Childhood trauma levels were not significantly different between the three groups (ANOVA $\mathrm{F}(1,91)=0.079, p$-value $=0.78)$. Also, no effect of time was present for SKA2 methylation (paired $t$-test, $t=-0.944, \mathrm{df}=92$, $p$-value $=0.348$ ).

To examine the effects of traumatic stress and PTSD per se, we also conducted longitudinal stratified analyses in nonPTSD participants and trauma-exposed individuals, separately (see Figure 1). Exposure to traumatic stress during deployment was associated with an increase in SKA2 methylation in individuals that did not develop PTSD symptoms after deployment $(B=0.005, t=4.195$, $p$-value $=$ $9.47 \times 10^{-5}$. Moreover, decreases in SKA2 methylation was associated with the development of deployment-related PTSD symptoms in individuals exposed to traumatic stress (excluding the participants that had low trauma exposures; $B=-0.070, t=-4.298, p$-value $=6.7 \times 10^{-5}$ ).

Supplementary Tables S1 and S2 show the full models of the association of SKA2 methylation levels with traumatic stress exposure and PTSD symptoms for the combined as well as the stratified analysis. Exclusion of participants for who alcohol, smoking, or medication use changed during deployment retained the association of PTSD symptoms with longitudinal changes in SKA2 methylation for medication use $(B=-0.060, \quad t=-2.803, \quad p$-value $=0.008), \quad$ smoking $\left(B=-0.069, t=-4.182 p\right.$-value $\left.=1.1 \times 10^{-4}\right)$, and alcohol use $\left(B=-0.075 t=-4.886\right.$, $p$-value $\left.=1.0 \times 10^{-5}\right)$.

Increases in PTSD symptoms were positively correlated with increases of psychoneuroticism symptoms (SCL-90 total score, $r=0.5, p<0.001)$. As a result, increases in psychoneuroticism in individuals exposed to traumatic stress were also significantly associated with longitudinal changes in $S K A 2$ methylation $(B=-0.063, t=-2.073, p$-value $=0.043$, (model fit: $\mathrm{F}(2,55)=16.6, p<0.001, R^{2}=0.35$ ). In contrast, SKA2 methylation changes were not significantly associated with any of the SCL-90 subscales of depression, somatization, agoraphobia, and anxiety (data not shown).

\section{PTSD Prediction from SKA2}

Finally, we investigated the prediction of PTSD symptoms from the previously published SKA2 suicide predictor rule (Guintivano et al, 2014). We found that the suicide

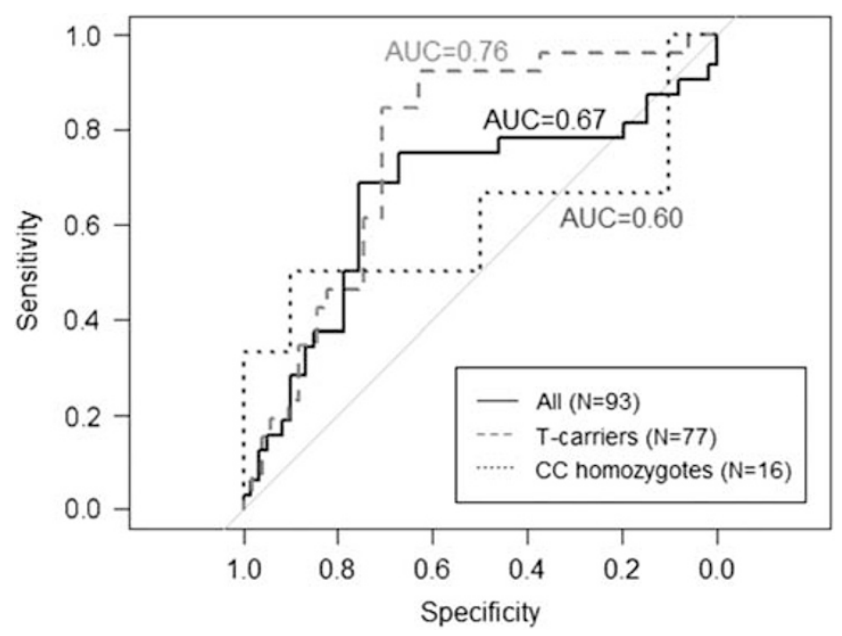

Figure 2 PTSD prediction using pre-deployment SKA2 methylation based on the previously published SKA2 prediction rule (Guintivano et al, 20I4).

prediction model provided modest but statistically significant prediction of PTSD from pre-deployment SKA2 methylation in a model including childhood trauma (AUC $=0.66,95 \%$ CI: $0.53-0.79$, $p$-value $=0.011$ ). Optimal sensitivity was 0.81 with a specificity of 0.90 . Permutation analysis showed that SKA2 methylation was a significantly stronger predictor than random CpGs (permuted $p$-value $=1.5 \mathrm{e}-4$ ). Subsequent stratified prediction analyses were carried out to identify the genotypes for which prediction was optimal, as SKA2 methylation is in part under genetic control. In T-allele carriers $(N=77)$, prediction was best $(\mathrm{AUC}=0.76,95 \% \mathrm{CI}$ : $0.65-0.87, p$-value $=7.0 \times 10^{-5}$ ) which yielded an optimal sensitivity of 0.96 with specificity of 0.94 . Prediction was lowest in CC homozygotes $(N=16$; AUC $=0.60,95 \%$ CI: $0.24-0.96, p$-value $=0.50$ ). Figure 2 shows the performance of the SKA2 prediction rule for PTSD for different genotypes groups. Even though the suicide prediction rule did not predict PTSD from baseline after exclusion of childhood trauma (AUC $=0.48,95 \%$ CI: $0.35-0.61$ ), prospective changes in SKA2 methylation during deployment in interaction with genotype resulted in a significant prediction in absence of childhood trauma as a factor (AUC $=0.69,95 \%$ CI:0.55-0.82), indicating some utility in complete absence of recorded history.

Training a new PTSD prediction rule for the development of PTSD symptoms from baseline SKA2 methylation $(N=93)$ showed that the development of PTSD symptoms is predicted fairly well in a model that included childhood trauma (AUC $=0.85,95 \% \mathrm{CI}: 0.76-0.92, p<0.001$ ), with an optimal sensitivity of 0.94 and a specificity of 0.70 . A model that also included pre-deployment PTSD symptoms was slightly better (AUC $=0.86,95 \%$ CI: 0.79-0.94) while prediction of PTSD symptoms from baseline SKA2 methylation levels in a model without childhood trauma yielded less robust results (AUC $=0.74,95 \% \mathrm{CI}$ : 0.64-0.84).

\section{DISCUSSION}

This study provides converging evidence for a role of SKA2 methylation in stress-related psychopathology. Longitudinal 
decreases in SKA2 methylation after deployment were related to the development of PTSD symptoms, whereas exposure to deployment-related traumatic stress was associated with increases in SKA2 methylation over time. In an independent sample, higher levels of SKA2 methylation were associated with a blunted cortisol stress response. These findings are consistent with an interpretation in which increases in SKA2 methylation after trauma reflects a functional adaptation of the physiological cortisol stress response. Conversely, unchanged or decreased SKA2 methylation may indicate persistence of a high-cortisol stress response with the risk of exhaustion of the HPA axis and subsequent development of PTSD. Such a role of SKA2 would be in agreement with its importance for HPA-axis functionality as highlighted by studies showing that SKA2 knockdown affects glucocorticoid feedback inhibition (Rice et al, 2008), and the association of (epi)genetic variation in SKA2 with suppression of the cortisol awakening response (Guintivano et al, 2014).

In addition to the longitudinal decrease in SKA2 methylation related to the development of PTSD symptoms, predeployment SKA2 methylation levels in concert with childhood trauma significantly predicted the development of PTSD symptoms. Validating a previously published SKA2 suicide prediction rule for PTSD using pre-deployment SKA2 methylation levels and childhood trauma resulted in a significant prediction of PTSD even when not taking trauma exposure during deployment into account (AUC: 0.66, $p$-value $=0.011)$. This prediction was particularly significant in T-allele carriers $(N=77, \mathrm{AUC}=0.76)$. The relevance of inclusion of childhood trauma in this model is in line with previous studies showing the effects of childhood trauma on HPA-axis activity (Yehuda et al, 1991; Carpenter et al, 2007; Lovallo et al, 2012; Heim et al, 2000). A new predictor rule derived from the baseline SKA2 methylation that again included childhood trauma, also predicted PTSD symptoms with fairly good accuracy (AUC $=0.85, p<0.001)$. These data suggest that SKA2 methylation together with childhood trauma may be of use as a PTSD biomarker.

The observation that the prediction is superior in T-allele carriers is difficult to interpret in light of the fact that DNA methylation changes occur on the C allele of the rs7208505 SNP. It is possible that the T-allele marks some relevant functional change through a mechanism that remains to be determined. Previous studies have demonstrated that SNPs within PTSD-associated genes may alter the threedimensional structure and facilitate interaction of enhancer and promoter regions to drive transcription (Klengel et al, 2013). A similar mechanism may be at play for SKA2 at rs7208505 or SNPs in linkage disequilibrium with this locus. Of relevance, publically available histone $\mathrm{H} 3$ lysine 27 acetylation and $\mathrm{H} 4$ tri-methylation signatures implicate a possible enhancer region within a few $\mathrm{kb}$ upstream of rs7208505 on chr17 within a HapMap implicated LD block with rs7208505. Alternatively, Guintivano et al (2014), originally reported an ENCODE implicated glucocorticoid receptor binding peak proximal to rs7208505 as well as one in the promoter region of SKA2. As these data were generated by immunoprecipitation for the glucocorticoid receptor following dexamethasone treatment, these two peaks may be an artifact of a single glucocorticoid receptor binding event occurring across three-dimensionally folded
DNA, further suggesting that genetic effects at rs7208505 may be mediating conformational changes to drive transcription of SKA2. However, these effects should be further investigated because at present they also may pose a chance finding in a small sample.

One particular strength of the current study is the possibility to study SKA2 methylation in a longitudinal design before and after deployment. To our knowledge, the SKA2 biomarker in this longitudinal study represents the first genetic and epigenetic biomarker with potential for prospective prediction of PTSD. Association of the SKA2 methylation levels with the endocrine stress response in an experimental stress paradigm suggests that SKA2 indicates the actual and functional response to stress, which adds to the credibility of the SKA2 biomarker. In contrast to the documented strong influence of gender on cortisol stress responsivity that is thought to reflect physiological differences (Vinkers et al, 2015b), the absence of a direct mediating effect of SKA2 methylation levels for cortisol stress reactivity suggests that SKA2 may function as a stress responsivity biomarker rather than a direct causal link. SKA2 methylation does not explain (mediate) the effects of childhood trauma on stress responsivity. The association of SKA2 methylation with several other HPA-axis related symptoms such as depression, neuroticism and suicide is consistent with the central role of stress-induced alterations in HPA-axis reactivity in PTSD and many other disorders (Mehta and Binder, 2012; De Kloet et al, 2006; Yehuda et al, 1991) and points to a role of SKA2 methylation as a general stress responsivity biomarker.

The results of this study should be interpreted in the context of its limitations. The longitudinal cohort included relatively small pre-selected groups, which precludes a meaningful analysis of positive predictive values. In addition, our findings were obtained in male Caucasian individuals and it may therefore be difficult to directly extrapolate the findings to other samples and populations.

In conclusion, this longitudinal study shows that traumatic stress and PTSD have opposite effects on SKA2 methylation. Whereas traumatic stress exposure leads to increasing SKA2 methylation levels, the development of PTSD is associated with decreasing SKA2 methylation. The fair prediction of PTSD from pre-deployment SKA2 methylation levels in concert with childhood trauma as well as the association with HPA-axis responsivity underscore the potential of SKA2 prediction. Together they provide a strong basis for further studies of genetic and epigenetic variation of SKA2 as a marker for stress susceptibility in general and for PTSD in particular.

\section{FUNDING AND DISCLOSURE}

Dr Kaminsky is an inventor on patent applications for DNA methylation biomarker based prediction of post-partum depression (PPD), suicide, and PTSD. The PPD technology is under option to Physician's Choice Laboratory Services. Dr Kaminsky received consultant fees from Janssen Research and Development, LLC. Dr Boks is an inventor on patent applications for DNA methylation biomarker based prediction of PTSD. The remaining authors declare no conflict of interest. 


\section{ACKNOWLEDGMENTS}

The data collection was funded by the Dutch Ministry of Defence. Methylation analyses were funded by the VENI Award fellowship from the Netherlands Organisation for Scientific Research (NWO, grant number 916.11.086) to BPFR. Funders had no role in design and reporting of the study.

\section{REFERENCES}

Bernstein DP, Stein JA, Newcomb MD, Walker E, Pogge D, Ahluvalia $\mathrm{T}$ et al (2003). Development and validation of a brief screening version of the Childhood Trauma Questionnaire. Child Abuse Negl 27: 169-190.

Boks MP, Schipper M, Schubart CD, Sommer IE, Kahn RS, Ophoff RA (2007). Investigating gene environment interaction in complex diseases: increasing power by selective sampling for environmental exposure. Int J Epidemiol 36: 1363-1369.

Boks MP, van Mierlo HC, Rutten BP, Radstake TR, de Wit L, Geuze E et al (2015). Longitudinal changes of telomere length and epigenetic age related to traumatic stress and post-traumatic stress disorder. Psychoneuroendocrinology 51: 506-512.

Bremner JD, Bolus R, Mayer EA (2007). Psychometric properties of the Early Trauma Inventory-Self Report. J Nerv Ment Dis 195: 211-218.

Carpenter LL, Carvalho JP, Tyrka AR, Wier LM, Mello AF, Mello MF et al (2007). Decreased adrenocorticotropic hormone and cortisol responses to stress in healthy adults reporting significant childhood maltreatment. Biol Psychiatry 62: 1080-1087.

De Kloet CS, Vermetten E, Geuze E, Kavelaars A, Heijnen CJ, Westenberg HG (2006). Assessment of HPA-axis function in posttraumatic stress disorder: pharmacological and non-pharmacological challenge tests, a review. J Psychiatr Res 40: 550-567.

Derogatis LR, Lipman RS, Covi L (1973). SCL-90: an outpatient psychiatric rating scale-preliminary report. Psychopharmacol Bull 9: $13-28$.

Guintivano J, Brown T, Newcomer A, Jones M, Cox O, Maher BS et al (2014). Identification and replication of a combined epigenetic and genetic biomarker predicting suicide and suicidal behaviors. Am J Psychiatry 171: 1287-1296.

Heim C, Newport DJ, Miller AH, Nemeroff CB (2000). Long-term neuroendocrine effects of childhood maltreatment. JAMA 284: 2321.

Houtepen LC, Boks MP, Kahn RS, Joels M, Vinkers CH (2015). Antipsychotic use is associated with a blunted cortisol stress response: a study in euthymic bipolar disorder patients and their unaffected siblings. Eur Neuropsychopharmacol 25: 77-84.

Hovens JE, Ramsen I, van der Ploeg HM (2000). Selfreport Measure For PTSD Symptoms: SIP Manual. Swets \& Zeitlinger: Leiden, The Netherlands.

Hovens JE, Bramsen I, van der Ploeg HM (2002). Self-rating inventory for posttraumatic stress disorder: review of the psychometric properties of a new brief Dutch screening instrument. Percept Mot Skills 94: 996-1008.

Jaffe AE, Irizarry RA (2014). Accounting for cellular heterogeneity is critical in epigenome-wide association studies. Genome Biol 15: R31.

Keane TM, Caddell JM, Taylor KL (1988). Mississippi scale for combat-related posttraumatic stress disorder: three studies in reliability and validity. J Consult Clin Psychol 56: 85-90.

Klengel T, Mehta D, Anacker C, Rex-Haffner M, Pruessner JC, Pariante CM et al (2013). Allele-specific FKBP5 DNA demethylation mediates gene-childhood trauma interactions. Nat Neurosci 16: 33-41.

Lovallo WR, Farag NH, Sorocco KH, Cohoon AJ, Vincent AS (2012). Lifetime adversity leads to blunted stress axis reactivity: studies from the Oklahoma Family Health Patterns Project. Biol Psychiatry 71: 344-349.
Malan-Muller S, Seedat S, Hemmings SM (2014). Understanding posttraumatic stress disorder: insights from the methylome. Genes Brain Behav 13: 52-68.

Mehta D, Binder EB (2012). Gene x environment vulnerability factors for PTSD: the HPA-axis. Neuropharmacology 62: 654-662.

Pruessner JC, Kirschbaum C, Meinlschmid G, Hellhammer DH (2003). Two formulas for computation of the area under the curve represent measures of total hormone concentration versus timedependent change. Psychoneuroendocrinology 28: 916-931.

Reijnen A, Rademaker AR, Vermetten E, Geuze E (2015). Prevalence of mental health symptoms in Dutch military personnel returning from deployment to Afghanistan: a 2-year longitudinal analysis. Eur Psychiatry 30: 341-346.

Rice L, Waters CE, Eccles J, Garside H, Sommer P, Kay P et al (2008). Identification and functional analysis of SKA2 interaction with the glucocorticoid receptor. J Endocrinol 198: 499-509.

Sandweiss DA, Slymen DJ, Leardmann CA, Smith B, White MR, Boyko EJ et al (2011). Preinjury psychiatric status, injury severity, and postdeployment posttraumatic stress disorder. Arch Gen Psychiatry 68: 496-504.

Schoenbaum M, Kessler RC, Gilman SE, Colpe LJ, Heeringa SG, Stein MB et al (2014). Predictors of suicide and accident death in the Army Study to Assess Risk and Resilience in Servicemembers (Army STARRS): results from the Army Study to Assess Risk and Resilience in Servicemembers (Army STARRS). JAMA Psychiatry 71: 493-503.

Schubeler D (2015). Function and information content of DNA methylation. Nature 517: 321-326.

Sundin J, Fear NT, Iversen A, Rona RJ, Wessely S (2010). PTSD after deployment to Iraq: conflicting rates, conflicting claims. Psychol Med 40: 367-382.

Thombs BD, Bernstein DP, Lobbestael J, Arntz A (2009). A validation study of the Dutch Childhood Trauma QuestionnaireShort Form: factor structure, reliability, and known-groups validity. Child Abuse Negl 33: 518-523.

Van Zuiden M, Geuze E, Maas M, Vermetten E, Heijnen CJ, Kavelaars A (2009). Deployment-related severe fatigue with depressive symptoms is associated with increased glucocorticoid binding to peripheral blood mononuclear cells. Brain Behav Immun 23: 1132-1139.

Van Zuiden M, Geuze E, Willemen HL, Vermetten E, Maas M, Heijnen CJ et al (2011). Pre-existing high glucocorticoid receptor number predicting development of posttraumatic stress symptoms after military deployment. Am J Psychiatry 168: 89-96.

Vinkers CH, Zorn JV, Cornelisse S, Koot S, Houtepen LC, Olivier B et al (2013). Time-dependent changes in altruistic punishment following stress. Psychoneuroendocrinology 38: 1467-1475.

Vinkers CH, Kalafateli AL, Rutten BP, Kas MJ, Kaminsky Z, Turner JD et al (2015a). Traumatic stress and human DNA methylation: a critical review. Epigenomics 7: 593-608.

Vinkers CH, Joëls M, Milaneschi Y, Gerritsen L, Kahn RS, Penninx BW et al (2015b). Mineralocorticoid receptor haplotypes sex-dependently moderate depression susceptibility following childhood maltreatment. Psychoneuroendocrinology 54: 90-102.

Witteveen AB, Van der Ploeg E, Bramsen I, Huizink AC, Slottje P, Smid T et al (2006). Dimensionality of the posttraumatic stress response among police officers and fire fighters: an evaluation of two self-report scales. Psychiatry Res 141: 213-228.

Yehuda R, Giller EL, Southwick SM, Lowy MT, Mason JW (1991). Hypothalamic-pituitary-adrenal dysfunction in posttraumatic stress disorder. Biol Psychiatry 30: 1031-1048.

Zovkic IB, Meadows JP, Kaas GA, Sweatt JD (2013). Interindividual Variability in Stress Susceptibility: A Role for Epigenetic Mechanisms in PTSD. Front Psychiatry 4: 60. 